TRANSACTIONS OF THE

AMERICAN MATHEMATICAL SOCIETY

Volume 351, Number 8, Pages 3197-3216

S 0002-9947(99)02324-7

Article electronically published on March 29, 1999

\title{
BILIPSCHITZ HOMOGENEOUS JORDAN CURVES
}

\author{
MANOUCHEHR GHAMSARI AND DAVID A. HERRON \\ Dedicated to Professor Frederick W. Gehring
}

\begin{abstract}
We characterize bilipschitz homogeneous Jordan curves by utilizing quasihomogeneous parameterizations. We verify that rectifiable bilipschitz homogeneous Jordan curves satisfy a chordarc condition. We exhibit numerous examples including a bilipschitz homogeneous quasicircle which has lower Hausdorff density zero. We examine homeomorphisms between Jordan curves.
\end{abstract}

\section{INTRODUCTION}

A space $X$ is homogeneous with respect to a family $\mathcal{F}$ of self-homeomorphisms of $X$ if the action of $\mathcal{F}$ on $X$ is transitive. In this paper we are primarily interested in the situation where $X=C$ is a Jordan curve, often in Euclidean space $\mathbf{R}^{n}$, and $\mathcal{F}$ is a family of bilipschitz homeomorphisms. In particular, we want to distinguish between the situation where $\mathcal{F}$ comprises self-maps of some ambient space versus the case when the homeomorphisms in $\mathcal{F}$ are defined only on $X$. In the latter case, when a metric space $X$ is homogeneous with respect to the family $\mathcal{B} \mathcal{L}_{K}(X)$ of all $K$-bilipschitz self-maps of $X$, we declare $X$ to be $K$-bilipschitz homogeneous, abbreviated $K-\mathrm{BLH}$; thus for each pair of points $x, y \in X$ there is a $K$-bilipschitz homeomorphism $f: X \rightarrow X$ satisfying $f(x)=y$.

Since homogeneity with respect to $\mathcal{B} \mathcal{L}_{K}\left(\mathbf{R}^{2}\right)$ implies quasiconformal homogeneity, a result due to Erkama [E1] asserts that these curves are quasicircles. Erkama's proof is based on compactness. Here we present a direct proof which provides an explicit estimate for the bounded turning constant. (See Section 2 for basic definitions, terminology and notation.)

Theorem A. Suppose $C \subset \mathbf{R}^{2}$ is a Jordan curve which is homogeneous with respect to $\mathcal{B} \mathcal{L}_{K}\left(\mathbf{R}^{2}\right)$. Then $C$ is bounded turning, hence a quasicircle, with constant $a=1+2 K^{2}$.

Example 3.1 illustrates that the exponent 2 is essentially best possible.

Theorem A suggests several questions. First, does the weaker condition of just homogeneity with respect to $\mathcal{B L}_{K}(C)$ imply that $C$ is bounded turning (a quasicircle in $\mathbf{R}^{2}$ )? Next, what is the situation in $\mathbf{R}^{n}$ ? We believe the following qualitative result warrants attention.

Received by the editors September 13, 1996 and, in revised form, December 15, 1997.

1991 Mathematics Subject Classification. Primary 30C65; Secondary 28A80.

Key words and phrases. Homogeneity, self-similarity, bilipschitz, bounded turning, quasicircle, Hausdorff measure, quasiconformal, fractal.

The second author was partially supported by the Charles Phelps Taft Memorial Fund at UC.

(C)1999 American Mathematical Society 
Theorem B. Let $C$ be a rectifiable Jordan curve in a metric space. Then $C$ is bilipschitz homogeneous if and only if $C$ satisfies a chordarc condition.

The proof of Theorem B reveals that a $K-\mathrm{BLH}$ rectifiable Jordan curve $C$ satisfies a uniform local chordarc condition with constant $K^{4} \ell(C) / \operatorname{diam}(C)$. However, as indicated by Example 4.2, to get a global chordarc condition we must consider the geometry of $C$. Theorem B provides no information for non-rectifiable curves, and there are plenty of non-rectifiable bilipschitz homogeneous curves including some with dimension one; see Example 5.3. Also, note that according to Theorem E, the $\alpha$-dimensional analog of Theorem B is false: There are bilipschitz homogeneous $\mathcal{H}^{\alpha}$-rectifiable curves which do not satisfy an $\alpha$-dimensional chordarc condition.

Now we turn to the problem of determining conditions which guarantee that a curve is bilipschitz homogeneous. It is easy to see, by conjugating rotations of $\mathbf{S}^{1}$, that $C$ is $K-\mathrm{BLH}$ if there is a homeomorphism $h: \mathbf{S}^{1} \rightarrow C$ which satisfies

$$
|h x-h y| \leq K|h u-h v| \text { when }|x-y| \leq|u-v|
$$

for all $x, y, u, v \in \mathbf{S}^{1}$. (See Proposition 4.1.) Theorem $\mathrm{C}$ indicates when this simple sufficient condition is also necessary for bilipschitz homogeneity.

We call embeddings $h: X \rightarrow Y$ which satisfy the above inequality weakly quasihomogeneous, abbreviated WQH. Since the inverse of a WQH map may not be WQH, we consider the following more general class of maps. We call an embedding $h: X \rightarrow Y$ quasihomogeneous, or $\mathrm{QH}$, provided

$$
\frac{|h x-h y|}{|h u-h v|} \leq \eta\left(\frac{|x-y|}{|u-v|}\right)
$$

for all distinct points $x, y, u, v$; here $\eta:[0,+\infty) \rightarrow[0,+\infty)$ is a homeomorphism, and we abbreviate this by saying that $h$ is $\eta-\mathrm{QH}$.

Quasihomogeneous maps form the natural class of homeomorphisms to study bilipschitz homogeneity. Again by conjugation we easily deduce that quasihomogeneous maps preserve bilipschitz homogeneity: If $h: X \rightarrow Y$ is an $\eta-\mathrm{QH}$ map of metric spaces and $X$ is homogeneous with respect to $\mathcal{B L}_{K}(X)$, then $Y$ is homogeneous with respect to $\mathcal{B L}_{\eta(K)}(Y)$; see Lemma 4.4. Here is additional information regarding these maps.

Theorem C. A Jordan curve $C \subset \mathbf{R}^{n}$ with positive finite Hausdorff measure is bilipschitz homogeneous and bounded turning if and only if $C=h\left(\mathbf{S}^{1}\right)$ for some $\eta-Q H$ homeomorphism $h: \mathbf{S}^{1} \rightarrow C$. Moreover, $\eta$ and the constants for $C$ depend only on each other and $n$.

Note that a consequence of Theorem $\mathrm{C}$ is that we actually have homogeneity with respect to a (compact abelian one-parameter) group of bilipschitz self-homeomorphisms. We mention that Mayer [My] has characterized Jordan plane curves which have weakly quasihomogeneous parameterizations as being orbits of oneparameter uniformly bilipschitz groups acting on $\mathbf{R}^{2}$. (We give a simple proof that plane Jordan curves with quasihomogeneous parameterizations are homogeneous with respect to $\mathcal{B} \mathcal{L}_{K}\left(\mathbf{R}^{2}\right)$; see Proposition 4.8.) An upshot of this and Theorem $\mathrm{C}$ is that bilipschitz homogeneous quasicircles with positive finite Hausdorff measure are homogeneous with respect to $\mathcal{B} \mathcal{L}_{K}\left(\mathbf{R}^{2}\right)$.

A natural question arises: When is a homeomorphism of bilipschitz homogeneous spaces quasihomogeneous? We verify this in the following special situation. Here we declare a homeomorphism $h: X \rightarrow Y$ to be quasi-measure preserving if $X, Y$ 
are $\alpha$-, $\beta$-sets respectively and there exists a constant $M$ such that for all $\alpha$-sets $A \subset X, \mathcal{H}^{\alpha}(A) / M \leq \mathcal{H}^{\beta}(f A) \leq M \mathcal{H}^{\alpha}(A)$.

Theorem D. A homeomorphism of bilipschitz homogeneous bounded turning Jordan curves in Euclidean spaces which have positive finite Hausdorff measures is quasi-measure preserving if and only if it is quasihomogeneous.

Our interest in bilipschitz homogeneity grew out of work in a previous paper $[\mathrm{GH}]$ where we studied $\alpha$-dimensional Ahlfors regular and chordarc conditions. It is easy to see that curves which satisfy an $\alpha$-dimensional chordarc condition are bilipschitz homogeneous, and bilipschitz homogeneous curves with positive lower density must satisfy an $\alpha$-dimensional Ahlfors regular condition; see [GH, Theorems B,F]. The necessity of requiring positive lower density came as somewhat of a surprise; we thank Pertti Mattila for directing our attention to the following; this example also demonstrates that many of our results are best possible - e.g., see Section 6.

Theorem E. There exist bilipschitz homogeneous quasicircles which have positive finite Hausdorff measure but lower Hausdorff density zero everywhere.

This paper is organized as follows. Section 2 contains definitions, terminology and other preliminary data. We establish Theorems A and B in Section 3. We study quasihomogeneous maps in Section 4 and substantiate Theorem C. Section 5 contains numerous examples which illustrate our results including a generalized snowflake construction verifying Theorem E. We examine homeomorphisms between Jordan curves in Section 6 and corroborate Theorem D there. We conclude with a list of questions in Section 7.

\section{Preliminaries}

Our notation is relatively standard. We let $B(x ; r)=\{y:|x-y|<r\}$ and $S(x ; r)=\{y:|x-y|=r\}$ denote the open ball and sphere of radius $r$ centered at the point $x$. We write $c=c(a, \ldots)$ to indicate a constant $c$ which depends only on the parameters $a, \ldots$ We write $a \approx b$ to mean there exists a positive finite constant $c$ with $a / c \leq b \leq a c$; typically $c$ will depend on various parameters, and we try to make this as clear as possible often giving explicit values.

Let $C$ be a Jordan curve; thus $C$ is a simple, closed, curve, i.e., a homeomorphic image of the unit circle $\mathbf{S}^{1}$. Given two points $x, y$ in $C$ we let $C(x, y)$ and $C^{\alpha}(x, y)$ denote the components of $C \backslash\{x, y\}$ with minimal diameter and minimal $\mathcal{H}^{\alpha}$-measure respectively. (Abusing notation we often assume that $C(x, y)$ or $C^{\alpha}(x, y)$ include the endpoints $x, y$.) Here $\mathcal{H}^{\alpha}$ denotes $\alpha$-dimensional Hausdorff measure which is defined by

$$
\mathcal{H}^{\alpha}(A)=\lim _{r \rightarrow 0}\left[\inf \left\{\sum_{1}^{\infty} \operatorname{diam}\left(U_{i}\right)^{\alpha}: A \subset \bigcup_{1}^{\infty} U_{i}, \operatorname{diam}\left(U_{i}\right) \leq r\right\}\right] .
$$

We often write $\ell$ for $\mathcal{H}^{1}$. We call $A$ an $\alpha$-set provided $0<\mathcal{H}^{\alpha}(A)<\infty$. The Hausdorff dimension of $A$ is

$$
\operatorname{dim}_{\mathcal{H}}(A)=\inf \left\{\alpha>0: \mathcal{H}^{\alpha}(A)<\infty\right\}=\sup \left\{\alpha>0: \mathcal{H}^{\alpha}(A)>0\right\} .
$$

The upper and lower $\mathcal{H}^{\alpha}$-densities of $A$ at $x$ are

$$
\bar{D}^{\alpha}(A, x)=\limsup _{r \rightarrow 0} \frac{\mathcal{H}^{\alpha}(A \cap B(x ; r))}{(2 r)^{\alpha}}, \quad \underline{D}^{\alpha}(A, x)=\liminf _{r \rightarrow 0} \frac{\mathcal{H}^{\alpha}(A \cap B(x ; r))}{(2 r)^{\alpha}} .
$$

For further details we refer to $[\mathrm{F} 1],[\mathrm{F} 2],[\mathrm{Mt}]$. 
Along with the quasi-measure preserving maps defined in the introduction, we make extensive use of Hausdorff measure preserving maps. Let $A, B$ be $\alpha$-, $\beta$-sets respectively. We call $f: A \rightarrow B$ an $\mathcal{H}^{\alpha} / \mathcal{H}^{\beta}$-measure preserving map provided $\mathcal{H}^{\alpha}(A) \mathcal{H}^{\beta}(f E)=\mathcal{H}^{\beta}(B) \mathcal{H}^{\alpha}(E)$ for all $\alpha$-sets $E \subset A$. It is easy to verify the following, which describes the curves which have ' $\mathcal{H}^{\alpha}$-parameterizations'.

2.1. Fact. Let $C$ be a Jordan curve. There exists an $\mathcal{H}^{1} / \mathcal{H}^{\alpha}$-measure preserving homeomorphism from $\mathbf{S}^{1}$ onto $C$ if and only if each non-degenerate subarc of $C$ is an $\alpha$-set.

Recall that $f$ is $K$-bilipschitz, abbreviated $K$-BL, if

$$
K^{-1}|x-y| \leq|f(x)-f(y)| \leq K|x-y| \text { for all } x, y .
$$

Next, we say that a map $f$ is $K-\mathrm{BL}^{\alpha}$ if

$$
|x-y| / K \leq|f(x)-f(y)|^{\alpha} \leq K|x-y| \text { for all } x, y .
$$

(Observe that this notation does not agree exactly with that in [GH].)

Of course even bilipschitz homeomorphisms generally fail to preserve measure. Thus we require information about how $\mathrm{BL}, \mathrm{BL}^{\alpha}$, and other maps distort Hausdorff measure. Minor modifications in the proof of $[\mathrm{F} 1,1.8, \mathrm{p} .10]$ yields the following which describes the change in Hausdorff measure due to a Hölder map.

2.2. Lemma. Suppose $f: X \rightarrow Y$ is surjective and $|f(x)-f(y)| \leq M|x-y|^{s}$ for all $x, y \in X$. Then

$$
\mathcal{H}^{\beta}(Y) \leq M^{\beta} \mathcal{H}^{s \beta}(X) \text { for all } \beta>0,
$$

and thus $\operatorname{dim}_{\mathcal{H}}(X) \geq s \operatorname{dim}_{\mathcal{H}}(Y)$.

2.3. Corollary. Suppose there is a $K-B L^{\alpha}$ homeomorphism from $X$ onto $Y$. Then $\beta=\operatorname{dim}_{\mathcal{H}}(Y)=\alpha \operatorname{dim}_{\mathcal{H}}(X)=\alpha \gamma$ and $K^{-\gamma} \mathcal{H}^{\gamma}(X) \leq \mathcal{H}^{\beta}(Y) \leq K^{\gamma} \mathcal{H}^{\gamma}(X)$.

A quasicircle is the image of $\mathbf{S}^{1}$ via a global quasiconformal homeomorphism of the plane. Ahlfors characterized quasicircles as the Jordan plane curves which satisfy a bounded turning condition [LV, §8.7, p.100]; that is, a Jordan curve $C \subset \mathbf{R}^{2}$ is a quasicircle if and only if there is a constant $a$ such that for all $x, y \in C$ we have

$$
\operatorname{diam}(C(x, y)) \leq a|x-y| .
$$

In the sequel we say that a Jordan curve $C \subset \mathbf{R}^{n}$ is $a-\mathrm{BT}$ if the above condition holds for all points $x, y \in C$. A related concept is described by saying that $C$ is chordarc, or CA, if $C$ is locally rectifiable and there is a constant $b$ such that for each pair of points $x, y \in C$

$$
\ell\left(C^{1}(x, y)\right) \leq b|x-y| .
$$

We abbreviate this by saying that $C$ is $b-\mathrm{CA}$. Since $\operatorname{diam} C(x, y) \leq \operatorname{diam} C^{1}(x, y) \leq$ $\ell\left(C^{1}(x, y)\right)$, it is immediate that chordarc curves are bounded turning (hence quasicircles in $\mathbf{R}^{2}$ ).

Now we list some elementary facts about bilipschitz homogeneity. Notice that for a BLH $C$ with $\operatorname{dim}_{\mathcal{H}}(C)=\alpha$, we have three mutually distinct possibilities for the measure of all non-trivial subarcs $A$ of $C$ : either $\mathcal{H}^{\alpha}(A)=0$ or $A$ is an $\alpha$-set or $\mathcal{H}^{\alpha}(A)=\infty$. Examples 5.1 and 5.3 illustrate that each of these situations do occur. A straightforward argument based on connectedness corroborates the following. 
2.4. Lemma. Let $C$ be a bilipschitz homogeneous Jordan curve. Then $\mathcal{H}^{\alpha}(C)>0$ if and only if $\mathcal{H}^{\alpha}(A)>0$ for each non-degenerate subarc $A$ of $C$. Thus $C$ is an $\alpha$-set if and only if each such subarc $A$ is an $\alpha$-set.

Notice that a consequence of Fact 2.1 and Lemma 2.4 is that BLH Jordan curves which are $\alpha$-sets have ' $\mathcal{H}^{\alpha}$-parameterizations'.

Any three distinct points $x, y, z$ on a Jordan curve $C$ induce an orientation on $C$ given by 'from $x$ through $y$ to $z$ '. Also, a self-homeomorphism $f$ of $C$ has the property that all images of such triples induce the same orientation, and thus it makes sense to call $f$ orientation preserving or reversing depending on whether or not the triple $f(x), f(y), f(z)$ induces the same orientation as $x, y, z$.

2.5. Lemma. Let $C$ be a $K-B L H$ Jordan curve. Then for each $x, y \in C$ there is an orientation preserving $K^{2}-B L f: C \rightarrow C$ with $f(x)=y$.

Proof. Fix $x_{0}, y_{0} \in C$ and let $f_{0}: C \rightarrow C$ be $K-\mathrm{BL}$ with $f_{0}\left(x_{0}\right)=y_{0}$. Assume $f_{0}$ is orientation reversing, and that there is a maximal subarc $A \subset C$ about $y_{0}$ such that for each $y \in A$ there exist only orientation reversing $K-$ BL self-homeomorphisms of $C$ mapping $x_{0}$ to $y$. Note that $A$ must be open. Suppose there is some point $y \in A$ with the property that there exits an orientation reversing $K-\mathrm{BL} g: C \rightarrow C$ with $g\left(y_{0}\right)=y$. Choose a $K-\mathrm{BL} h: C \rightarrow C$ with $h\left(x_{0}\right)=y$. Since $h$ is orientation reversing, $f=g^{-1} \circ h$ is an orientation preserving $K^{2}$-BL self-map of $C$ with $f\left(x_{0}\right)=y_{0}$.

Now assume that for all points $y \in A$ there exist only orientation preserving $K$-BL self-homeomorphisms of $C$ mapping $y_{0}$ to $y$. Suppose $A$ is closed. Then by connectedness, $A=C$. Thus $x_{0} \in A$, so $f=g^{-1}$ is orientation preserving $K-\mathrm{BL}$ with $f\left(x_{0}\right)=y_{0}$, where $g: C \rightarrow C$ with $g\left(y_{0}\right)=x_{0}$. Finally, assume that $A$ is not closed. Thus there are points $y_{i} \in A$ with $y_{i} \rightarrow z \notin A$. Choose $K-\mathrm{BL} g_{i}: C \rightarrow C$ with $g_{i}\left(y_{0}\right)=y_{i}$. Then $g=\lim g_{i_{k}}: C \rightarrow C$ is orientation preserving and $K-\mathrm{BL}$ with $g\left(y_{0}\right)=z$. Since $z \notin A$, there is an orientation preserving $K-\mathrm{BL} h: C \rightarrow C$ with $h\left(x_{0}\right)=z$. Then $f=g^{-1} \circ h: C \rightarrow C$ is $K^{2}-\mathrm{BL}$, orientation preserving and satisfies $f\left(x_{0}\right)=y_{0}$.

Notice that when $C$ is homogeneous with respect to a (closed) group of $K-\mathrm{BL}$ homeomorphisms, then the above argument shows that we can assume that each map is orientation preserving.

\section{Bounded Turning and Chord-Arc Conditions}

In this section we exhibit several examples of bilipschitz homogeneous curves and we prove Theorems A, B. We begin by examining bilipschitz homogeneity in the plane. We first illustrate why the BT constant exhibited in Theorem A is essentially best possible.

3.1. Example. Assume $a \geq 1$ and let $C$ be the rectangle $C=([-a, a] \times\{0,1\}) \cup$ $(\{-a, a\} \times[0,1])$ in $\mathbf{R}^{2}$. If there is a $K-B L$ self-homeomorphism of $C$ which maps the point $i=(0,1)$ to $z=(a, 0)$, then $K^{2} \geq \sqrt{1+a^{2}}$.

Proof. Let $f: C \rightarrow C$ be $K-$ BL with $z=f(i)$. Put $w=f(0)$ and choose $\zeta=z$ or $\zeta=-z$ so that $f(\zeta)$ lies on $C(z, w)$. Then

$$
K \geq|f(i)-f(0)|=|z-w| \geq|z-f(\zeta)| \geq|i-\zeta| / K .
$$


Proof of Theorem A. Fix $x, y \in C$. Assume $\alpha \cap C=\{x, y\}$ where $\alpha=[x, y]$. Let $G$ be the interior of $\alpha \cup C(x, y)$ and let $D$ be the component of the complement of $C$ containing $G$. We work with the distance function

$$
\lambda_{D}(z, w)=\inf \{\ell(\gamma): \gamma \text { joins } z, w \text { in } D\} .
$$

It is not difficult to demonstrate that $\lambda_{D}(z, \alpha) \leq b|x-y|$ for all $z \in A$ implies $\operatorname{diam}(A) \leq(1+2 b)|x-y|$, where $A$ can be either of the components of $C \backslash\{x, y\}$. Choose $z \in C(x, y)$ with

$$
L=\lambda_{D}(z, \alpha)=\sup \left\{\lambda_{D}(\zeta, \alpha): \zeta \in C(x, y)\right\} .
$$

We verify that $L \leq K^{2}|x-y|$, which in turn implies that $C$ is $a-\mathrm{BT}$ with $a=$ $1+2 K^{2}$.

Select a $K$-BL self-homeomorphism $f$ of $\mathbf{R}^{2}$ with $f(C)=C$ and $f(x)=z$. Since $C$ is bounded, $f(D)=D$. If $\alpha \cap f(\alpha)=\emptyset$, then $f(\alpha)$ joins $z$ to $\alpha$, whence

$$
L \leq \ell(f \alpha) \leq K \ell(\alpha)=K|x-y| .
$$

Suppose $\alpha \cap f(\alpha) \neq \emptyset$. There are two cases depending on whether or not $f(G) \subset$ $G$. Assume $f(G) \subset G$, and let $\zeta=f(z)$. Now choose an $\operatorname{arc} \beta \subset \bar{D}$ with $\lambda_{D}(\zeta, \alpha)=$ $\ell(\beta)$. Since $f(\alpha)$ separates $\zeta$ from $\alpha$ in $D, \beta \cap f(\alpha) \neq \emptyset$. Let $\beta^{\prime}, \beta^{\prime \prime}$ be the components of $\beta \backslash f(\alpha)$ which join $f(\alpha)$ and $\zeta, \alpha$ respectively. Then

$$
\lambda_{D}(\zeta, f \alpha) \leq \ell\left(\beta^{\prime}\right), \quad \lambda_{D}(\alpha, f \alpha) \leq \ell\left(\beta^{\prime \prime}\right)
$$

and

$$
\ell\left(\beta^{\prime}\right)+\ell\left(\beta^{\prime \prime}\right) \leq \ell(\beta) \leq L \leq \ell(f \alpha)+\lambda_{D}(\alpha, f \alpha) \leq \ell(f \alpha)+\ell\left(\beta^{\prime \prime}\right)
$$

so

$$
L \leq K \lambda_{D}(\zeta, f \alpha) \leq K \ell\left(\beta^{\prime}\right) \leq K \ell(f \alpha) \leq K^{2}|x-y| .
$$

Finally, if $f(G) \not \subset G$, we argue as above with $\zeta=f(w)$, where $w$ is an arbitrary point of $C \backslash C(x, y)$, to see that $\lambda_{D}(w, \alpha) \leq K^{2}|x-y|$; this then yields $\operatorname{diam}(C(x, y)) \leq \operatorname{diam}(C \backslash C(x, y)) \leq\left(1+2 K^{2}\right)|x-y|$.

Further information regarding quasiconformal homogeneity can be found in the works of MacManus, Näkki and Palka [MNP], Gehring and Palka [GP], Erkama [E1],[E2], and Sarvas [Sa]. In fact a weaker definition for homogeneity with respect to $\mathcal{B} \mathcal{L}_{K}\left(\mathbf{R}^{2}\right)$ can be adopted, as observed by Erkema and Brechner [BE],[E2]. For example, if $C$ is a non-degenerate proper subcontinuum of $\mathbf{S}^{2}$ and if for each pair of points $x, y \in C$ there exists an open $U \supset C$ and a $K$-bilipschitz $f: U \rightarrow f(U)$ with $f(C)=C$ and $f(x)=y$, then $C$ is a bilipschitz homogeneous closed Jordan curve. Indeed, as in [BE], [E2] we find that $C$ is a quasicircle, and results of Tukia and Väisälä [TV2, 2.18, 2.19(b)] asserts that $f: C \rightarrow C$ has a bilipschitz extension to $\mathbf{S}^{2}$ and hence $C$ is homogeneous with respect to $\mathcal{B} \mathcal{L}_{K}\left(\mathbf{R}^{2}\right)$.

According to Example 4.2, there is no hope in estimating a BT constant solely in terms of $K$ for $K-\mathrm{BLH}$ curves in $\mathbf{R}^{n}$. On the other hand, we see from Theorem $\mathrm{B}$ that every rectifiable bilipschitz homogeneous curve must be bounded turning .

Proof of Theorem B. Let $C$ be a rectifiable Jordan curve. If $C$ is $b-\mathrm{CA}$, it is easy to see that arclength motion along $C$ is $b-\mathrm{BL}$ and hence $C$ is $b-\mathrm{BLH}$. Now suppose that $C$ is $\sqrt{K}-\mathrm{BLH}$. Put $\delta=\operatorname{diam}(C) / 2 K$. Examining an arclength parameterization of $C$ (which is bi-uniformly continuous) we obtain an $\varepsilon=\varepsilon(\delta, C)>$ 
0 such that $\ell(C(x, y)) \geq \delta$ implies $|x-y| \geq \varepsilon$; for such $x, y$ the chordarc condition holds with $b=\ell(C) / \varepsilon$. Thus it suffices to consider $x, y \in C$ with $|x-y|<\varepsilon$, in which case $\ell(C(x, y))<\delta$.

Fix points $p, q \in C$ with $|p-q|=\operatorname{diam}(C)$. Let $A$ be a component of $C \backslash\{p, q\}$ with $\ell(A) \leq \ell(C) / 2$. Assume $(p, A, q)$ has the same orientation as $(x, C(x, y), y)$. According to Lemma 2.5 we can select a $K-$ BL $f_{1}: C \rightarrow C$ with $x_{1}=f_{1}(x)=p$. Note that $y_{1}=f_{1}(y) \in A$ and $q \notin A_{1}=f_{1}(C(x, y)) \subset A$, because otherwise we would have

$$
\operatorname{diam}(C)=|p-q| \leq \operatorname{diam}\left(A_{1}\right) \leq K \operatorname{diam}(C(x, y)) \leq K \ell(C(x, y))
$$

which would imply that $\ell(C(x, y))>\delta$. Continue this process by choosing $K-\mathrm{BL}$ $f_{i}: C \rightarrow C$ with $x_{i}=f_{i}(x)=y_{i-1}$ and $y_{i}=f_{i}(y) \in A \backslash\left(A_{1} \cup \cdots \cup A_{i-1}\right)$ until we get $q \in A_{N+1}\left(\right.$ here $\left.A_{i}=f_{i}(C(x, y))\right)$. Then $A_{1} \cup \cdots \cup A_{N} \subset A \subset A_{1} \cup \cdots \cup A_{N+1}$ and so

$$
\ell(A) \geq \sum_{1}^{N} \ell\left(A_{i}\right) \geq(N / K) \ell(C(x, y)) .
$$

We claim that $N \geq \delta /|x-y|$. We have

$$
\left|q-y_{N}\right| \leq \ell\left(A_{N+1}\right) \leq K \ell(C(x, y)) \leq K \delta
$$

so

$$
\left|p-y_{N}\right| \geq|p-q|-\left|q-y_{N}\right| \geq \operatorname{diam}(C)-K \delta=\operatorname{diam}(C) / 2
$$

whence

$$
\operatorname{diam}(C) / 2 \leq\left|p-y_{N}\right| \leq \sum_{1}^{N}\left|x_{i}-y_{i}\right| \leq N K|x-y|,
$$

or $N \geq \delta /|x-y|$ as asserted. Thus

$$
\ell(A) \geq(N / K) \ell(C(x, y)) \geq \frac{\delta}{K} \frac{\ell(C(x, y))}{|x-y|}
$$

and we obtain

$$
\ell(C(x, y)) \leq a|x-y|
$$

where $a=K \ell(A) / \delta \leq K^{2} \ell(C) / \operatorname{diam}(C)$. It now follows that $C$ is $b-\mathrm{CA}$ with $b=\max \{a, \ell(C) / \varepsilon\}$.

We mention that there are plenty of bilipschitz homogeneous Jordan curves which fail to be rectifiable. Indeed, the well-known von Koch snowflakes are $K-\mathrm{BLH}$ where $K$ depends only on the Hausdorff dimension. (For the reader's convenience we discuss these curves in Example 5.1; see also [GH, 5.2].) Moreover, there are even non-rectifiable BLH curves with dimension one; see Example 5.3. Also, we point out that the $\alpha$-dimensional analog of Theorem $\mathrm{B}$ is false: Theorem $\mathrm{E}$ provides a BLH Jordan curve which is a $\beta$-set, hence $\mathcal{H}^{\beta}$-rectifiable, but it is not $\beta$-dimensional chordarc because it has lower $\mathcal{H}^{\beta}$-density zero everywhere. 


\section{Quasihomogeneous Maps}

In this section we discuss some elementary properties of quasihomogeneous homeomorphisms and we prove Theorem 4.6 (which gives Theorem $\mathrm{C}$ as a corollary) and Proposition 4.8. We begin with the following idea which is established by conjugating rotations of the circle. What is interesting is that this simple sufficient condition is actually necessary for bilipschitz homogeneity when our space is a BT Jordan curve, with positive finite Hausdorff measure, in any metric space.

4.1. Proposition. Let $C$ be a Jordan curve in a metric space and suppose there is a homeomorphism $\varphi: \mathbf{S}^{1} \rightarrow C$ which satisfies

$$
|\varphi(z)-\varphi(w)| \leq K|\varphi u-\varphi v| \text { when } \quad|z-w|=|u-v| .
$$

Then $C$ is $K-B L H$ with respect to a compact one-parameter abelian group.

Now we demonstrate that there are $K-\mathrm{BLH}$ curves, with $K$ fixed, which have arbitrarily large BT constants. Moreover, these curves illustrate a surprising phenomenon: The maps $\varphi$ described in Proposition 4.1 need not be $K-\mathrm{WQH}$ !

4.2. Example. For each $0<\lambda<1 / 10$ there exists a Jordan curve $C$ in $\mathbf{R}^{3}$ which is 3-bilipschitz homogeneous, however any bounded turning constant for $C$ is greater than $1 / \lambda$. In fact, there is a homeomorphism $\varphi: \mathbf{S}^{1} \rightarrow C \subset \mathbf{R}^{3}$ which satisfies

$$
|\varphi(z)-\varphi(w)| \leq 3|\varphi(u)-\varphi(v)| \text { when }|z-w|=|u-v|,
$$

however any WQH constant for $\varphi$ exceeds $1 / 2 \lambda$.

Proof. Fix $0<\lambda \leq 1 / 10$. Define $F(t)=(x(t), y(t), z(t))$ for $0 \leq t \leq 2 \pi$ where

$$
x(t)=\cos (2 t)(1-\lambda \cos (t)), \quad y(t)=\sin (2 t)(1-\lambda \cos (t)), \quad z(t)=\lambda \sin (t) .
$$

Then $C=F([0,2 \pi])$ is a Jordan curve in $\mathbf{R}^{3}$ which can be visualized as a wiggly 'figure-eight' folded back on itself. As $|F(0)-F(\pi)|=2 \lambda$ and diam $C(F(0), F(\pi)) \geq$ $2, C$ can be $a-\mathrm{BT}$ only when $a \geq 1 / \lambda$. It suffices now to verify that $\varphi\left(e^{i t}\right)=F(t)$ satisfies the asserted inequalities. For this it is enough to substantiate that

$$
3^{-1}|F(t)-F(s)| \leq|F(t+x)-F(s+x)| \leq 3|F(t)-F(s)|
$$

for all $t, s, x$. Here we have extended the domain of $F$ to all of $\mathbf{R}$.

A straightforward, although tedious, calculation leads to

$$
\begin{aligned}
|F(t)-F(s)|^{2} & =2[1-\cos (2 t-2 s)][1-\lambda(\cos t+\cos s)] \\
& +2 \lambda^{2}[1-\sin t \sin s-\cos t \cos s \cos (2 t-2 s)] \\
& =4 \sin ^{2}(t-s)[1-\lambda(\cos t+\cos s)] \\
& +4 \lambda^{2}\left[\sin ^{2}((t-s) / 2)+\cos t \cos s \sin ^{2}(t-s)\right] .
\end{aligned}
$$

Putting $\theta=(t-s) / 2$ and using the double angle formula for $\sin 2 \theta$ yields

$$
\begin{gathered}
4 \sin ^{2} \theta\left(3 \cos ^{2} \theta+\lambda^{2}\left[1+4 \cos ^{2} \theta \cos t \cos s\right]\right) \leq|F(t)-F(s)|^{2} \\
\leq 4 \sin ^{2} \theta\left(5 \cos ^{2} \theta+\lambda^{2}\left[1+4 \cos ^{2} \theta \cos t \cos s\right]\right) .
\end{gathered}
$$

The desired inequalities follow from these when we replace $t, s$ by $t+x, s+x$ and consider the cases $|\cos \theta| \leq 1 / 4$ and $|\cos \theta| \geq 1 / 4$. 
In attempting to replace the circle $\mathbf{S}^{1}$ in Proposition 4.1 by an arbitrary BLH space we encounter a difficulty. This led us to the class of quasihomogeneous maps as defined in the Introduction; these homeomorphisms seem to have been studied first by Shalaginov; see [Sh], [AS]. This class is preserved under the operations of taking inverses and composition. Trivially, $\mathrm{BL}$ and $\mathrm{BL}^{\alpha}$ maps are $\mathrm{QH}$ which in turn are QS (quasisymmetric). In particular, quasihomogeneous images of BT sets are BT. Section 6 contains further information concerning QH homeomorphisms.

Obviously every $\eta-\mathrm{QH}$ map is $\eta(1)-\mathrm{WQH}$. Mimicking the proof of [V, 2.9] (or [TV1, 2.15]) due to Väisälä (and Tukia), it is not difficult to verify that in many situations a weakly QH homeomorphism is in fact quasihomogeneous. For our purposes we record this as follows.

4.3. Fact. Let $X \subset \mathbf{R}^{n}$ and $Y \subset \mathbf{R}^{m}$. Suppose $X$ is path-connected. Then every $K-W Q H$ homeomorphism from $X$ to $Y$ is $\eta-Q H$ where $\eta$ depends only on $K, n, m$.

We remind the reader of our belief that quasihomogeneous maps form the natural class of homeomorphisms to study bilipschitz homogeneity.

4.4. Lemma. If $h: X \rightarrow Y$ is an $\eta$-quasihomogeneous homeomorphism of metric spaces and $X$ is $K-B L H$, then $Y$ is $\eta(K)-B L H$.

Proof. Given $y_{i}=h\left(x_{i}\right) \in Y$, we define $F=h \circ f \circ h^{-1}$ where $f: X \rightarrow X$ is $K-\mathrm{BL}$ with $f\left(x_{1}\right)=x_{2}$. Then $F: Y \rightarrow Y$ is $\eta(K)-\mathrm{BL}$ and $F\left(y_{1}\right)=y_{2}$.

In a previous paper we examined the following $\mathcal{H}^{\alpha} /$ diam inequalities:

$$
(\operatorname{diam}(A))^{\alpha} / b \leq \mathcal{H}^{\alpha}(A) \leq b(\operatorname{diam}(A))^{\alpha} .
$$

For example, a BT Jordan curve $C$ satisfies the above for all subarcs $A \subset C$ if and only if $C=\varphi\left(\mathbf{S}^{1}\right)$ for some $\mathrm{BL}^{\alpha}$ homeomorphism $\varphi: \mathbf{S}^{1} \rightarrow C$ if and only if $C$ satisfies an $\alpha$-dimensional chordarc condition. There are 'nice' Jordan curves which satisfy the above inequalities but fail to be BT; see [GH, Theorem B, Example 5.5] $\left(\mathrm{BL}^{\alpha}\right.$ here corresponds to $\mathrm{BL}^{1 / \alpha}$ in $\left.[\mathrm{GH}]\right)$. Here we are interested in the following weaker versions of the above inequalities. We consider a Jordan curve $C$ and ask when are there constants $b, c$ such that for all subarcs $A, B$ of $C$ either

$$
\mathcal{H}^{\alpha}(A) \leq \mathcal{H}^{\alpha}(B) \quad \text { implies } \quad \operatorname{diam}(A) \leq b \operatorname{diam}(B)
$$

or

$$
\operatorname{diam}(A) \leq \operatorname{diam}(B) \quad \text { implies } \quad \mathcal{H}^{\alpha}(A) \leq c \mathcal{H}^{\alpha}(B)
$$

It turns out that when $C$ is a BLH $\alpha$-set, (HD) holds and if in addition $C$ is $\mathrm{BT},(\mathrm{DH})$ holds (at least in nice metric spaces). (Of course these conditions are meaningless when $C$ is not an $\alpha$-set.) The former assertion is a straightforward consequence of an elementary packing argument.

4.5. Lemma. Let $C$ be a Jordan curve (in any metric space) which is a $\sqrt{K}-B L H$ $\alpha$-set. Suppose $A, B$ are subarcs of $C$. Then

$$
\mathcal{H}^{\alpha}(A) \leq c \mathcal{H}^{\alpha}(B) \quad \text { implies } \quad \operatorname{diam}(A) \leq K\left(1+c K^{\alpha}\right) \operatorname{diam}(B) .
$$

Proof. Let $x, y$ and $z, w$ be the 'first, last' endpoints of $B$ and $A$ respectively. By Lemma 2.5 we can select a $K-$ BL map $f_{1}: C \rightarrow C$ with $f_{1}(x)=z$ and so that $A$ and $B_{1}=f_{1}(B)$ 'overlap'. If $A \subset B_{1}$, then $\operatorname{diam}(A) \leq \operatorname{diam}\left(B_{1}\right) \leq K \operatorname{diam}(B)$ and we are done. 
Suppose that $A \not \subset B_{1}$. Put $z_{1}=f_{1}(y)$. Choose a $K-$ BL map $f_{2}: C \rightarrow C$ with $f_{2}(x)=z_{1}$ so that $B_{1}$ and $B_{2}=f_{2}(B)$ are adjacent. If $A \subset B_{1} \cup B_{2}$, then $\operatorname{diam}(A) \leq 2 K \operatorname{diam}(B)$ and we are done (consider the case $k=1$ below).

Suppose $A \not \subset B_{1} \cup B_{2}$ and that we have continued the above process to obtain adjacent $\operatorname{arcs} B_{i}=f_{i}(B) \subset A$ where $f_{i}: C \rightarrow C$ is $K-\mathrm{BL}(i=1, \ldots, k)$. Then

$$
c \mathcal{H}^{\alpha}(B) \geq \mathcal{H}^{\alpha}(A) \geq \sum_{i=1}^{k} \mathcal{H}^{\alpha}\left(B_{i}\right) \geq k \mathcal{H}^{\alpha}(B) / K^{\alpha}
$$

Since $B$ is an $\alpha$-set we obtain $k \leq c K^{\alpha}$. This means that the above process terminates -i.e., we get $A \subset B_{1} \cup \cdots \cup B_{k}$-as soon as $k>c K^{\alpha}$. Letting $k$ be the smallest integer larger than $c K^{\alpha}$ we obtain

$$
\operatorname{diam}(A) \leq k K \operatorname{diam}(B) \leq K\left(1+c K^{\alpha}\right) \operatorname{diam}(B) .
$$

Now we substantiate a list of equivalent conditions for bilipschitz homogeneity. As mentioned in the Introduction, Theorem $\mathrm{C}$ is an immediate corollary.

4.6. Theorem. For a Jordan curve $C$ (in any metric space) with positive finite Hausdorff measure, the following are equivalent.

(a) $C$ is $K-B L H$ and $a-B T$.

(b) $C$ is $a-B T$ and (HD) holds.

(c) $C=\varphi\left(\mathbf{S}^{1}\right)$ where $\varphi: \mathbf{S}^{1} \rightarrow C$ is $H-W Q H$.

The constants depend only on each other as indicated in the proof. Moreover, if $C \subset \mathbf{R}^{n}$, then the above are also equivalent to each of the following.

(d) $C=\varphi\left(\mathbf{S}^{1}\right)$ where $\varphi: \mathbf{S}^{1} \rightarrow C$ is $\eta-Q H$.

(e) $C$ is $a-B T$ and (HD), (DH) both hold.

Proof. Let $C$ be a Jordan curve which is an $\alpha$-set. If $C$ is $K-\mathrm{BLH}$, then Lemma 4.5 asserts that $C$ satisfies (HD) with $b=K^{2}\left(1+K^{2 \alpha}\right)$. Thus (a) implies (b). When (c) is assumed: Proposition 4.1 asserts that $C$ is $K-\mathrm{BLH}$ with $K=H$, and it is easy to see that $C$ must be $a-\mathrm{BT}$ with $a=H$; thus (a) holds. We certify that (b) implies (c).

Suppose that $C$ is $a-\mathrm{BT}$ and that (HD) holds for subarcs $A, B$ of $C$. An obvious consequence of (HD) is that each non-degenerate subarc of $C$ is an $\alpha$-set. Thus according to Fact 2.1, there is an $\mathcal{H}^{\alpha}$-parameterization $\varphi: \mathbf{S}^{1} \rightarrow C$. We assume $\mathcal{H}^{\alpha}(C)=2 \pi$. We demonstrate that $\varphi$ is weakly quasihomogeneous.

Fix points $z_{i}, w_{i} \in \mathbf{S}^{1}$ with $\left|z_{1}-z_{2}\right| \leq\left|w_{1}-w_{2}\right|$. Let $I, J$ be the shorter subarcs of $\mathbf{S}^{1}$ joining $z_{1}$ to $z_{2}, w_{1}$ to $w_{2}$ respectively and put $x_{i}=\varphi\left(z_{i}\right), y_{i}=\varphi\left(w_{i}\right)$. As $\ell(I) \leq \ell(J)$, we have

$$
\mathcal{H}^{\alpha}\left(C^{\alpha}\left(x_{1}, x_{2}\right)\right)=\mathcal{H}^{\alpha}(\varphi(I)) \leq \mathcal{H}^{\alpha}(\varphi(J))=\mathcal{H}^{\alpha}\left(C^{\alpha}\left(y_{1}, y_{2}\right)\right) \leq \mathcal{H}^{\alpha}\left(C\left(y_{1}, y_{2}\right)\right) .
$$

Appealing to (HD) and the fact that $C$ is $a-\mathrm{BT}$ we thus obtain

$$
\left|x_{1}-x_{2}\right| \leq \operatorname{diam} C^{\alpha}\left(x_{1}, x_{2}\right) \leq b \operatorname{diam} C\left(y_{1}, y_{2}\right) \leq a b\left|y_{1}-y_{2}\right| .
$$

We conclude that $\varphi$ is $a b-\mathrm{WQH}$.

Now suppose that $C \subset \mathbf{R}^{n}$. Appealing to Fact 4.3 we see that (c) implies (d) with $\eta$ depending only on $H$ and $n$. Obviously (e) implies (b) and thus it remains to demonstrate that (d) implies (e).

We may assume that $\varphi: \mathbf{S}^{1} \rightarrow C$ is an $\eta-\mathrm{QH} \mathcal{H}^{\alpha}$-parameterization. Fix subarcs $A, B$ of $C$ with $\operatorname{diam}(A) \leq \operatorname{diam}(B)$. Choose $I, J \subset \mathbf{S}^{1}$ with $A=\varphi(I), B=\varphi(J)$. 
We show that $\operatorname{diam}(I) \leq c \operatorname{diam}(J)$, so $\ell(I) \leq c \ell(J)$, hence $\mathcal{H}^{\alpha}(A) \leq c \mathcal{H}^{\beta}(B)$ and thus (DH) holds.

We may assume that both $\mathcal{H}^{\alpha}(A)$ and $\mathcal{H}^{\alpha}(B)$ are at most $(1 / 2) \mathcal{H}^{\alpha}(C)$; if $A$ fails to satisfy this condition, then we replace $A$ with $\varphi\left(I^{\prime}\right)$ where $I^{\prime}$ is half of $I$, while if $B$ is big we are done. Thus both of the $\operatorname{arcs} I$ and $J$ lie in semi-circles.

Let $x_{i}=\varphi\left(z_{i}\right)$ and $y_{i}=\varphi\left(w_{i}\right)$ be the endpoints of $A$ and $B$ respectively (so $z_{i}, w_{i}$ are the endpoints of $\left.I, J\right)$. Notice that if $\operatorname{diam}(B)=\left|\varphi\left(u_{1}\right)-\varphi\left(u_{2}\right)\right|$ with $u_{i} \in J$, then $\left|u_{1}-u_{2}\right| \leq\left|w_{1}-w_{2}\right|$ and thus $\operatorname{diam}(B) \leq H\left|y_{1}-y_{2}\right|, H=\eta(1)$.

Next, assume that $\operatorname{diam}(I)>\operatorname{diam}(J)$. Write $I=I_{0} \cup \bigcup_{1}^{k} J_{i}$ where $J_{i}$ are adjacent subarcs of $I$ which are congruent to $J$ and $\operatorname{diam}\left(I_{0}\right)<\operatorname{diam}(J)$. For $i=1, \ldots, k$ let $\zeta_{i-1}, \zeta_{i}$ be the endpoints of $J_{i}$, where $\zeta_{0}=z_{1}$. Then

$$
\left|\zeta_{0}-\zeta_{k}\right| \leq k\left|\zeta_{0}-\zeta_{1}\right|=k\left|w_{1}-w_{2}\right|
$$

so $\left|w_{1}-w_{2}\right|=(\pi / 2 k)\left|z_{1}-\zeta_{k}\right|$ and thus $\left|y_{1}-y_{2}\right| \leq \eta(\pi / 2 k)\left|x_{1}-\varphi\left(\zeta_{k}\right)\right|$. Therefore

$$
\begin{aligned}
\operatorname{diam}(B) & \geq \operatorname{diam}(A) \geq\left|x_{1}-\varphi\left(\zeta_{k}\right)\right| \geq(1 / \eta(\pi / 2 k))\left|y_{1}-y_{2}\right| \\
& \geq(1 / H \eta(\pi / 2 k)) \operatorname{diam}(B)
\end{aligned}
$$

which yields $k \leq \pi / 2 \eta^{-1}(1 / H)$. Letting $c=k+1$ we obtain $\ell(I) \leq c \ell(J)$ and $\mathcal{H}^{\alpha}(A) \leq c \mathcal{H}^{\alpha}(B)$ as desired.

4.7. Corollary. Let $C$ be a Jordan curve which is an $\alpha$-set. If $C$ is $K-B L H$ and $a-B T$, then the $\mathcal{H}^{\alpha}$-parameterization of $C$ is $a K^{2}\left(1+K^{2 \alpha}\right)-W Q H$.

We conclude this section with a simple proof of the following; cf. [My].

4.8. Proposition. A plane Jordan curve which has a weakly quasihomogeneous parameterization is homogeneous with respect to $\mathcal{B} \mathcal{L}_{K}\left(\mathbf{R}^{2}\right)$ for some fixed $K$.

Proof. Let $\varphi: \mathbf{S}^{1} \rightarrow C \subset \mathbf{R}^{2}$ be WQH. Employing [G, 2.8] we extend $\varphi$ to a QC $\Phi: \mathbf{R}^{2} \rightarrow \mathbf{R}^{2}$ with $\Phi$ BL with respect to the quasihyperbolic metrics in each component of $\mathbf{R}^{2} \backslash \mathbf{S}^{1}$. Fix points $x, y \in C$. As in the proof of Proposition 4.1, we obtain a BL $f: C \rightarrow C$ with $f(x)=y$ by setting $f=\varphi \circ \rho \circ \varphi^{-1}$ where $\rho: \mathbf{S}^{1} \rightarrow \mathbf{S}^{1}$ is the rotation from $\varphi^{-1}(x)$ to $\varphi^{-1}(y)$. Then $F=\Phi \circ \rho \circ \Phi^{-1}: \mathbf{R}^{2} \rightarrow \mathbf{R}^{2}$ satisfies $\left.F\right|_{C}=f$ which is BL, $F(C)=C$ and $F(x)=y$. Also, $F$ is BL with respect to the quasihyperbolic metrics in each component of $\mathbf{R}^{2} \backslash \mathbf{S}^{1}$. Therefore a result of Gehring's (see [G, 2.7]) asserts that $F$ is a BL self-map of $\mathbf{R}^{2}$. Note that we can get $\Phi(z)=z$ (hence $F(\zeta)=e^{i \theta} \zeta$ ) for all large $z(\zeta)$.

4.9. Corollary. A bilipschitz homogeneous quasicircle with positive finite Hausdorff measure is homogeneous with respect to $\mathcal{B L}_{K}\left(\mathbf{R}^{2}\right)$ for some fixed $K$.

\section{EXAMPLES}

Here we exhibit examples which illustrate the concepts presented throughout this paper. We begin with the familiar von Koch snowflakes; these are quasicircles homogeneous with respect to bilipschitz self-homeomorphisms of $\mathbf{R}^{2}$.

5.1. Example. For each $1 \leq \alpha<2$ there are $\alpha$-dimensional bilipschitz homogeneous curves.

Proof. Fix $\alpha \in[1,2)$ and choose $t \in(1 / 4,1 / 2)$ with $4 t^{\alpha}=1$. Define a sequence $\left\{K_{n}\right\}$ of Jordan arcs as follows. First, $K_{0}=[0,1]$. Next, $K_{1}=\bigcup_{k=1}^{4} I_{1}^{k}$ where $I_{1}^{k}=\sigma_{k}\left(K_{0}\right), \sigma_{k}$ is a similarity from $K_{0}$ onto the interval $\left[a_{k-1}, a_{k}\right]$, and (in complex 
notation) $a_{0}=0, a_{1}=t, a_{2}=1 / 2+i \sqrt{t-1 / 4}, a_{3}=1-t, a_{4}=1$. Then $K_{2}=$ $\bigcup_{k=1}^{4} I_{2}^{k}$ where $I_{2}^{k}=\sigma_{k}\left(K_{1}\right)$. Iterating this process yields a sequence of Jordan arcs $\left\{K_{n}\right\}$ with the property that $K_{n}$ converges (in the Hausdorff metric) to a von Koch snowflake arc which we denote by $K^{\alpha}[\mathrm{H}, \mathrm{pp} .728-729]$.

Employing Hutchinson's open set condition [H, 5.2, (1) and (2), pp.735-736] we find that $\operatorname{dim}_{\mathcal{H}}\left(K^{\alpha}\right)=\alpha$. A calculation shows that $\mathcal{H}^{\alpha}\left(K^{\alpha}\right)=1$. The 'natural' parameterization, given as the limit of the piecewise affine maps $[0,1] \rightarrow K_{n}$, is an $\mathcal{H}^{1} / \mathcal{H}^{\alpha}$-measure preserving $\mathrm{BL}^{\alpha}$ homeomorphism from $[0,1]$ to $K^{\alpha}$; see [T, (4), p.151]. We obtain a Jordan curve, the $\alpha$-dimensional von Koch snowflake $\mathbf{K}^{\alpha}$, by proceeding as above but starting with an equilateral triangle in place of the interval $[0,1]$ (the 'bumps' go outside the triangle).

The earnest reader can warm-up for the proof of Theorem $\mathrm{E}$ by verifying the following concerning the 'middle-third' von Koch snowflake. Note that (b) is essentially the fact that $\mathbf{K}^{\alpha}$ satisfies the $\mathcal{H}^{\alpha}$ / diam inequalities mentioned just after Lemma 4.4; this holds because $\mathbf{K}^{\alpha}$ is $\mathrm{CA}^{\alpha}$.

5.2. Fact. Let $\alpha=\log 4 / \log 3$. Then:

(a) $\mathbf{K}^{\alpha}$ is $4-B T$.

(b) If $U$ is a subarc of $K_{n}$ consisting of $N$ segments, then

$$
1 / 4 \leq N^{-1}\left(\operatorname{diam}(U) / 3^{-n}\right)^{\alpha} \leq 2^{\alpha+1} .
$$

While the von Koch procedure does not seem to generalize for the situation $n \geq 3$, it is possible to construct self-similar (in fact $\mathrm{CA}^{\alpha}$ ), hence bilipschitz homogeneous, Jordan curves having arbitrary dimension $\alpha \in[1, n)$; see [GH, 5.2].

Now we modify the von Koch snowflake construction. First we describe some bilipschitz homogeneous curves which fail to have positive or finite Hausdorff measure. Furthermore, each curve will be homogeneous with respect to $\mathcal{B} \mathcal{L}_{K}\left(\mathbf{R}^{2}\right)$.

5.3. Example. Given $1 \leq \alpha<2$ there is a bilipschitz homogeneous quasicircle $C$ in $\mathbf{R}^{2}$ with $\operatorname{dim}_{\mathcal{H}}(C)=\alpha$ but $\mathcal{H}^{\alpha}(A)=\infty$ for all non-degenerate subarcs $A \subset C$. Given $1<\alpha<2$ there is a bilipschitz homogeneous quasicircle $C$ in $\mathbf{R}^{2}$ with $\operatorname{dim}_{\mathcal{H}}(C)=\alpha$ but $\mathcal{H}^{\alpha}(A)=0$ for all subarcs $A \subset C$.

Proof. We modify the von Koch snowflake construction. Fix $1 \leq \alpha<2$ and $t \in[1 / 4,1 / 2)$ with $4 t^{\alpha}=1$. Choose $1 / 2>t_{1}>t_{2}>\cdots>t_{i} \searrow t$. (Here we choose $t_{i}$ decreasing slowly to $t$.) Now do the snowflake construction 'using $t_{i}$ ': At the $i^{\text {th }}$ stage we obtain a piecewise linear $\operatorname{arc} A_{i}$ consisting of $4^{i}$ line segments each of size $s_{i}=t_{i} t_{i-1} \ldots t_{2} t_{1}$. In the Hausdorff metric there is a limit arc $A$. Applying this process to each edge of an equilateral triangle yields a Jordan curve $C$.

Fix $\beta>\alpha$. Then $t_{i}^{\beta} \searrow t^{\beta}<t^{\alpha}=1 / 4$, so $4^{i} s_{i}^{\beta}=\left(4 t_{i}^{\beta}\right) \ldots\left(4 t_{1}^{\beta}\right) \rightarrow 0$. Thus considering the cover of $C$ by basic subarcs we find that $\mathcal{H}^{\beta}(C)=0$. Since this holds for all $\beta>\alpha, \operatorname{dim}_{\mathcal{H}}(C) \leq \alpha$. If $\alpha=1$, then since the dimension of any continuum is at least one, $\operatorname{dim}_{\mathcal{H}}(C)=1$. Suppose $\alpha>1$. Choose $1 \leq \beta<\alpha$. Then $t_{i}^{\beta} \searrow t^{\beta}>t^{\alpha}=1 / 4$, so $4^{i} s_{i}^{\beta}=\left(4 t_{i}^{\beta}\right) \ldots\left(4 t_{1}^{\beta}\right) \rightarrow \infty$. A standard argument now gives $\mathcal{H}^{\beta}(C)=\infty$; see [F2, pp.31-32] or [Mt, pp.61-62]. Thus $\operatorname{dim}_{\mathcal{H}}(C)=\alpha$.

Arguing as above we can show that each non-degenerate subarc of $C$ has infinite $\mathcal{H}^{\alpha}$-measure, or we can derive this from the fact that $C$ is BLH. That $C$ is a BLH quasicircle follows from the fact that $C$ has a $\mathrm{QH}$ parameterization. In fact, it is not difficult to see that the 'natural' parameterization, given by the limit of the piecewise affine maps $[0,1] \rightarrow A_{i}$, is weakly quasihomogeneous. 
The construction for the second group of examples is similar.

Below we furnish an example which has a surprising number of consequences; see Corollary 5.8 and Section 6 . We were led to this by our initial belief that bilipschitz homogeneous curves which were $\alpha$-sets would be $\alpha$-dimensional Ahlfors regular. We thank Pertti Mattila for suggesting this construction. A related example can be found in [My, pp.160-161]. We expect that one could use the ideas in [GH, 5.2] to construct such examples for dimensions $2 \leq \beta<n$.

Here is a sketch of our generalized snowflake construction. Given $1<\beta<2$, choose $\beta<\alpha<2$. Begin the usual snowflake construction as in Example 5.1 to get an $\alpha$-dimensional chordarc curve. At some point stop this $\alpha$-dimensional construction. We now have a piecewise linear polygonal curve with, say, $M$ straight line segments each of length $d$ and $M d^{\alpha}=1$.

Next, divide each of these $M$ line segments into equal parts all having length $\delta$ and so that $N \delta^{\beta}$ is bounded away from $0,+\infty$ by absolute constants; here $N=$ $M \cdot d / \delta$ is the total number of smaller segments. Now start the $\alpha$-dimensional construction again on each of these smaller segments. Continue this $\alpha$-dimensional construction longer than the first time, but eventually stop and do the "subdivision of each straight line segment" again.

Iterate the above process, each time continuing the $\alpha$-dimensional process longer and longer. Standard methods yield a limit Jordan curve $C$ and it is not too difficult to see that $C$ is a $\beta$-set. However, verifying other properties of $C$ requires some work. For the reader's benefit, and because of the importance of this example, we furnish the following detailed analysis.

Proof of Theorem E. First we explain the construction of the Jordan curve $C$. This is a mixing of two patterns: the familiar von Koch snowflake construction is interlaced with dividing each line segment into small equal pieces. We give details for the case $\beta=\operatorname{dim}_{\mathcal{H}}(C)=(1+\alpha) / 2$ where $\alpha=\log 4 / \log 3$, but our method generalizes. Let $K_{n}$ be the $n^{\text {th }}$ approximating arc for the 'middle-third' von Koch snowflake arc $K^{\alpha}$; see Example 5.1. Put $J_{1}=K_{1}$. For $i=1,2, \ldots$ we obtain $J_{i+1}$ from $J_{i}$ by dividing each line segment in $J_{i}$ into $3^{i}$ equally spaced subintervals and then replacing each of these subintervals with an appropriately scaled copy of $K_{i+1}$. We build a sequence of Jordan curves $\left\{C_{i}\right\}$ by beginning with an equilateral triangle and performing a similar construction. It is easy to see that $\left\{J_{i}\right\}$ (respectively, $\left\{C_{i}\right\}$ ) approaches a Jordan arc $J$ (respectively, a Jordan curve $C$ ) in the Hausdorff metric. Below we corroborate the following properties for $C$.

(a) $C$ is $4-\mathrm{BT}$,

(b) $1 / 4 \leq \mathcal{H}^{\beta}(C) \leq 3$,

(c) $\underline{D}^{\beta}(C, z)=0$ for each $z \in C$,

(d) $C$ is $K-\mathrm{BLH}$ for some constant $K$.

It is easy to see that the BT constant for $C$ is the same as that for the von Koch snowflake curve $\mathbf{K}^{\alpha}$, so Fact 5.2(a) yields (a).

Here is some terminology. A maximal straight line segment in $J_{i}$ is called an $i$-cell. We let $m_{i}$ be the number of $i$-cells $I$ in $J_{i}$ and put $d_{i}=\operatorname{diam}(I)$. (Notice that the Hausdorff distance between $J_{i}$ and $J_{k}$ is at most $d_{i}$ for all $k \geq i \geq 1$, and therefore $\left\{J_{i}\right\}$ is a Cauchy sequence in the Hausdorff metric.) Next, the contracted copies of $K_{i}$ used to construct $J_{i}$ are termed $i$-arcs (each consists of $4^{i} i$-cells). We let $n_{i}$ denote the total number of $i$-arcs $A$ in $J_{i}$ and write $\delta_{i}=\operatorname{diam}(A)$; so $\delta_{i}$ 
is the scaling factor used and each $i$-arc is congruent to $\delta_{i} K_{i}$. The endpoints of $i$-cells are called $i$-vertices and the endpoints of $i$-arcs are designated $i$-endpoints. Note that every $i$-vertex (so every $i$-endpoint) is an $(i+1)$-endpoint (thus also an $(i+1)$-vertex $)$.

Using this terminology we see that $J_{i+1}$ is obtained from $J_{i}$ by dividing each $i$-cell into $3^{i}$ segments of length $\delta_{i+1}$ and then replacing each of these segments by an $(i+1)$-arc. Thus, $J_{i}$ is a union of $n_{i} i$-arcs (copies of $\left.\delta_{i} K_{i}\right)$ and so consists of $m_{i}=4^{i} n_{i} i$-cells of size (length) $d_{i}=3^{-i} \delta_{i}=3^{i} \delta_{i+1}$. We have

$$
\delta_{i}=3^{-i(i-1)}, \quad n_{i}=12^{i(i-1) / 2}, \quad d_{i}=3^{-i^{2}}, \quad m_{i}=4^{i} 12^{i(i-1) / 2}, \quad n_{i} \delta_{i}^{\beta}=1 .
$$

The middle-third snowflake has dimension $\alpha$ because $4^{i}\left(3^{-i}\right)^{\alpha}=1$. The rightmost equality above is the analog of this for our generalized snowflake $C$, and is why $C$ has dimension $\beta$.

We interrupt our proof for some preliminary details. First we establish an analog of Fact 5.2(b) for subarcs of $J_{i}$. This is an essential ingredient in verifying both the measure properties and the bilipschitz homogeneity of $C$.

5.4. Lemma. Let $U$ be a subarc of $J_{i}$ which is a union of $N$ i-arcs. Suppose $\operatorname{diam}(U) \leq \delta_{i-1}$ (so $U$ does not properly contain an $(i-1)$-arc). Then

$$
N \delta_{i}^{\beta} \leq 12(\operatorname{diam} U)^{\beta} .
$$

Proof. Let $x, y$ be the endpoints of $U$; so $U=J_{i}(x, y)$ and $x, y$ are $i$-endpoints. We consider four cases depending on whether or not $x$ and $y$ are $(i-1)$-vertices. Suppose one of them is not an $(i-1)$-vertex, and there is not an $(i-1)$-vertex between them. In this situation $x$ and $y$ must belong to some $(i-1)$-cell, so $(\operatorname{diam} U)^{\beta}=\left(N \delta_{i}\right)^{\beta} \geq N \delta_{i}^{\beta}$.

Now suppose both $x$ and $y$ are $(i-1)$-vertices. First assume $x, y$ belong to some $(i-1)$-arc $A$. Then $N=3^{i-1} M$ where $M$ is the number of $(i-1)$-cells in $A$ between $x, y$. Since $A$ is congruent to $\delta_{i-1} K_{i-1}$ we see from Fact $5.2(\mathrm{~b})$ that

$$
N \leq 3^{i-1} 4\left(\operatorname{diam} U / 3^{-(i-1)} \delta_{i-1}\right)^{\alpha}
$$

This inequality in conjunction with $3^{\alpha}=4$ and $\operatorname{diam}(U) \leq \delta_{i-1}=9^{i-1} \delta_{i}$ yields

$$
N \delta_{i}^{\beta} \leq 4(\operatorname{diam} U)^{\beta} \text {. }
$$

Next assume $x, y$ do not belong to the same $(i-1)$-arc. Then they must lie on adjacent $(i-1)$-arcs. Let $z$ be the $(i-1)$-endpoint between $x$ and $y$. Letting $V$ be the closure of the larger of the two subarcs of $U \backslash\{z\}$, so $V$ contains $M \geq N / 2$ $i$-arcs, and using the preceding argument we obtain

$$
N \delta_{i}^{\beta} \leq 2 M \delta_{i}^{\beta} \leq 8(\operatorname{diam} V)^{\beta} \leq 8(\operatorname{diam} U)^{\beta} .
$$

Finally, suppose again that one of $x, y$ is not an $(i-1)$-vertex, and also that there is an $(i-1)$-vertex between them. Let $\xi, \eta$ be $(i-1)$-vertices in $U$ separating $x, y$ with $\xi, \eta$ closest to $x, y$ respectively (any of $x=\xi, \xi=\eta, \eta=y$ is possible). Let $U_{1}, U_{2}, U_{3}$ be the subarcs of $U$ with endpoints $x$ and $\xi, \xi$ and $\eta, \eta$ and $y$, and consisting of $N_{1}, N_{2}, N_{3} i$-arcs respectively. Now $x, \xi$ belong to some $(i-1)$-cell, and likewise for $y, \eta$. Thus by the very first case we have

$$
N_{j} \delta_{i}^{\beta} \leq\left(\operatorname{diam} U_{j}\right)^{\beta} \text { for } j=1,3 .
$$


Next, $\xi$ and $\eta$ belong to some $(i-1)$-arc, so by a previous case

$$
N_{2} \delta_{i}^{\beta} \leq 4\left(\operatorname{diam} U_{2}\right)^{\beta} .
$$

Choosing $j$ so that $N_{j} \geq N / 3$ we deduce that

$$
N \delta_{i}^{\beta} \leq 3 N_{j} \delta_{i}^{\beta} \leq 12\left(\operatorname{diam} U_{j}\right)^{\beta} \leq 12(\operatorname{diam} U)^{\beta} .
$$

5.5. Corollary. Let $U=J_{i}(x, y)$ be a union of $N$ i-arcs with $\operatorname{diam}(U) \leq \delta_{i-1}$. Fix $k>i$, put $V=J_{k}(x, y)$, and let $M$ be the number of $k$-arcs in $V$. Then

$$
M \delta_{k}^{\beta} \leq 12(\operatorname{diam} V)^{\beta} .
$$

Proof. $N / n_{i}=M / n_{k}$ and $n_{i} \delta_{i}^{\beta}=1$.

We say that $x, y$ and $z, w$ are equally spaced endpoints provided all four points are $i$-endpoints for some $i$ and $x, y$ and $z, w$ are separated by the same number of $i$-arcs. An easy counting argument furnishes the following.

5.6. Lemma. Let $0, z$ and $x, y$ be equally spaced $(i+1)$-endpoints. Let $\zeta$ be the $i$-endpoint just before or just after $z$. Then there are $i$-endpoints $\xi, \eta$ with the property that $0, \zeta$ and $\xi, \eta$ are equally spaced $i$-endpoints and $x, \xi$ (respectively, $y$, $\eta)$ belong to an $i$-arc.

The next result plays a crucial role in our proof that $C$ is bilipschitz homogeneous.

5.7. Lemma. There is an absolute constant $L_{0}$ such that if $0, z$ and $x, y$ are equally spaced $j$-endpoints with $z$ on the first or second $k$-cell or $k$-arc, then

$$
L_{0}^{-1}|x-y| \leq|z| \leq L_{0}|x-y| .
$$

Proof. Suppose $z$ lies on the first $k$-cell. Then $|z|$ is equal to the number of $(k+1)$ arcs between 0 and $z$ times $\delta_{k+1}$. Using bounded turning and Lemma 5.4 where necessary, it is straightforward to estimate $|x-y|$ by a similar quantity. When $z$ lies on the second $k$-cell the situation is even easier.

Now suppose $z$ lies on the first $k$-arc. We outline the argument for the case when $z, x, y$ are all $k$-vertices; the general case then follows by approximation. Assume $z, x, y$ are $k$-vertices and also that $x, y$ belong to the same $k$-arc. In this situation we appeal to Fact 5.2 and find that both $|z|^{\alpha}$ and $|x-y|^{\alpha}$ can be estimated by the number of $k$-cells between $0, z$ times $d_{k}^{\alpha}$. When $x, y$ belong to different $k$-arcs, there are three pictures to consider and we apply the above reasoning to $x, w$ and $y, w$ where $w$ is the $k$-endpoint between $x, y$.

Finally, suppose $z$ belongs to the second $k$-arc. Let $\zeta$ be the $k$-endpoint between $0, z$; consider $0, \zeta$ and $\zeta, z$ separately and argue as above.

Continuation of the proof of Theorem E. We demonstrate that $C$ enjoys the properties (b)-(d) listed just above Lemma 5.4. First, covering $J$ by $i$-arcs gives $\mathcal{H}_{\delta_{i}}^{\beta}(J)=n_{i} \delta_{i}^{\beta}=1$, so $\mathcal{H}^{\beta}(C)=3 \mathcal{H}^{\beta}(J) \leq 3$. For the opposite inequality in (b) we may assume, by compactness and an approximation argument, that $\left\{U_{k}\right\}$ is a finite cover of $J$ by subarcs, that each $U_{k}$ consists of a union of $N_{k} i$-arcs where $i$ is a (large) fixed integer independent of $k$, and that $\operatorname{diam}\left(U_{k}\right) \leq \delta_{i-1}$ for all $k$. Utilizing Lemma 5.4 and the fact that $\left\{U_{k}\right\}$ covers $J$ we deduce that

$$
\sum\left(\operatorname{diam} U_{k}\right)^{\beta} \geq(1 / 12) \sum N_{k} \delta_{i}^{\beta} \geq(1 / 12) n_{i} \delta_{i}^{\beta}=1 / 12 .
$$


Now we verify (c). Look at $A_{i}=J\left(0, d_{i}\right)$, a union of $3^{i}$ arcs each congruent to $B_{i}=J\left(0, \delta_{i+1}\right)$. As there are $n_{i+1}(i+1)$-arcs,

$$
\mathcal{H}^{\beta}\left(A_{i}\right)=3^{i} \mathcal{H}^{\beta}\left(B_{i}\right)=3^{i} \mathcal{H}^{\beta}(J) / n_{i+1} .
$$

Then since $n_{i+1} \delta_{i+1}^{\beta}=1$,

$$
\frac{\mathcal{H}^{\beta}\left(A_{i}\right)}{\left(2 d_{i}\right)^{\beta}}=\frac{3^{i} \mathcal{H}^{\beta}\left(B_{i}\right)}{2^{\beta}\left(3^{i} \delta_{i+1}\right)^{\beta}}=\frac{\mathcal{H}^{\beta}(J)}{2^{\beta}} 3^{(1-\beta) i} \rightarrow 0 \quad \text { as } i \rightarrow \infty,
$$

because $\beta>1$. Thus $\underline{D}^{\beta}(J, 0)=0$. Similarly, $C$ has lower density zero at the origin, and since $C$ is bilipschitz homogeneous (by (d)), this holds at every point.

Finally, we establish (d). According to Proposition 4.1 it suffices to find a WQH parameterization $\mathbf{S}^{1} \rightarrow C$; for this we need only consider the natural map $[0,1] \rightarrow J$ given as the limit of the piecewise-affine homeomorphisms $[0,1] \rightarrow J_{i}$. Since the $i$-endpoints are dense in $J$, it is enough to produce a constant $K$ such that whenever $x, y$ and $z, w$ are equally spaced endpoints, $|z-w| \leq K|x-y|$. In fact, it suffices to substantiate the existence of an absolute constant $L$ satisfying

$$
L^{-1}|x-y| \leq|z| \leq L|x-y|
$$

whenever $0, z$ and $x, y$ are equally spaced endpoints. (Thus self-homeomorphisms of $C_{i}$ which leave $i$-vertices invariant are bilipschitz on $i$-endpoints; hence limits of these maps will be BL self-homeomorphisms of $C$, and clearly $C$ is homogeneous with respect to the family of these limits.)

We use induction. It is easy to see that $(*)$ holds with $L=\sqrt{3}$ for 1-endpoints and with $L=2$ for 2 -endpoints. We verify that $(*)$ holds with $L=L_{i}$ for equally spaced $i$-endpoints where $L_{2}=L_{0}$ and $L_{i+1}=\left(1+3^{1-i}\right)\left(1+2 / 3^{i-1}\right) L_{i}$ for $i=2,3, \ldots$

Assume we have shown $(*)$ valid with $L=L_{i}$ for equally spaced $i$-endpoints. Let $0, z$ and $x, y$ be equally spaced $(i+1)$-endpoints. (Look at $i=3$.) We consider the various cases determined as $z$ 'moves' past the first $i$-arc, then past the first $(i-1)$-arc, and so forth until we reach the first (and only) 1 -arc. When $z$ belongs to either the first or second $i$-cell or $i$-arc in $J_{i}$, then Lemma 5.7 asserts that (*) is true with $L=L_{0}$. Thus all is OK at the ' $i$ 'th level'.

Now consider the ' $(i-1)^{\text {st }}$ level'. Let $z_{1}, x_{1}, y_{1}$ be $i$-endpoints closest to $z, x, y$ respectively with $z_{1}$ just before (or equal to) $z$ and with $0, z_{1}$ and $x_{1}, y_{1}$ separated by the same number of $i$-arcs. We claim first that if $z_{1}$ lies on either the first or second $(i-1)$-cell of $J_{i-1}$, then $(*)$ holds with $L=L_{0}$. To see this we let $w_{1}$ be the $(i+1)$-endpoint with $0, z_{1}$ and $x, w_{1}$ equally separated. Now think of $z_{1}, z$ as being $0, z-z_{1}$ and apply the proof of Lemma 5.7 to $0, z_{1}$ and to $z_{1}, z$.

Next suppose $z$ lies beyond the second $(i-1)$-cell of $J_{i-j-1}$, and $z_{1}$ is on the first $(i-1)$-arc of $J_{i-j-1}$. Now $0, z$ are separated by two $(i-1)$-cells, so $x, y$ and $x_{1}, y_{1}$ are separated by (at least) one $(i-1)$-cell and thus

$$
|z| \geq d_{i-1}, \quad\left|z_{1}\right| \geq d_{i-1}, \quad|x-y| \geq d_{i-1}, \quad\left|x_{1}-y_{1}\right| \geq d_{i-1}=3^{i-1} \delta_{i} .
$$

As $z, z_{1}$ and $x, x_{1}$ and $y, y_{1}$ belong to respective $i$-arcs, their respective distances from each other are at most $\delta_{i}$. Since $(*)$ holds for $z_{1}, x_{1}, y_{1}$ with $L=L_{i}$, we have

$$
|z| \leq\left(1+3^{1-i}\right)\left|z_{1}\right| \leq\left(1+3^{1-i}\right) L_{i}\left|x_{1}-y_{1}\right| \leq\left(1+3^{1-i}\right)\left(1+2 / 3^{i-1}\right) L_{i}|x-y|
$$

and similarly

$$
|x-y| \leq\left(1+3^{1-i}\right)\left(1+2 / 3^{i-1}\right) L_{i}|z| .
$$


Thus in both cases $(*)$ holds with $L=\left(1+3^{1-i}\right)\left(1+2 / 3^{i-1}\right) L_{i}=L_{i+1}$, and all is OK at the ' $(i-1)^{\text {st }}$ level'.

If $z$ (hence $z_{1}$ ) lies beyond the first $(i-1)$-arc of $J_{i-1}$, we continue this process replacing $i-1$ by $i-2$ and so forth. After the ' $(i-j)^{\text {th }}$ level' we get $(i-j)$ endpoints $z_{j+1}, x_{j+1}, y_{j+1}$ closest to $z_{j}, x_{j}, y_{j}$ respectively with $z_{j+1}$ just before (or equal to) $z_{j}$ and with $0, z_{j+1}$ and $x_{j+1}, y_{j+1}$ separated by the same number of $(i-j)$-arcs. If $z_{j+1}$ lies on either the first or second $(i-j-1)$-cell of $J_{i-j-1}$, then just as in the first case of the argument for the ' $(i-1)^{\text {st }}$ level' we see that $(*)$ holds for $z_{j}, x_{j}, y_{j}$ with $L=L_{0}$. Thus $(*)$ holds for $z_{j-1}, x_{j-1}, y_{j-1}$ with $L=\left(1+3^{j-i}\right)\left(1+2 / 3^{i-j}\right) L_{0}$ and eventually we see that $(*)$ holds for $z, x, y$ with $L=\left(1+3^{1-i}\right) \ldots\left(1+3^{j-i}\right)\left(1+2 / 3^{i-1}\right) \ldots\left(1+2 / 3^{i-j}\right) L_{0} \leq L_{i+1}$.

Next suppose $z$ lies beyond the second $(i-j-1)$-cell of $J_{i-j-1}$, and $z_{j+1}$ is on the first $(i-j-1)$-arc of $J_{i-j-1}$. We argue as in the latter part of the ' $(i-1)^{\text {st }}$ level': $(*)$ holds for $z_{j+1}, x_{j+1}, y_{j+1}$ with $L=L_{i-j}$, so by comparing $\left|z_{j+1}\right|$, $\left|x_{j+1}-y_{j+1}\right|$ to $\left|z_{j}\right|,\left|x_{j}-y_{j}\right| \ldots$ to $|z|,|x-y|$ we find that $(*)$ holds with $L=$ $\left(\prod_{1}^{j+1}\left(1+3^{k-i}\right)\left(1+2 / 3^{i-k}\right)\right) L_{i-j}=L_{i+1}$. Thus all is OK at the ' $(i-j-1)^{\text {st }}$ level'.

In the worst possible situation we continue the above process until we reach $j=i-1$, because then $z_{j}, x_{j}, y_{j}$ are 2-endpoints and $z_{j}$ is on the first and only 1 -arc.

5.8. Corollary. Let $C$ be the generalized snowflake constructed above. Then:

(a) The natural map $\mathbf{S}^{1} \rightarrow C$ is quasihomogeneous and $\mathcal{H}^{1} / \mathcal{H}^{\beta}$-measure preserving but not bilipschitz nor $B L^{\beta}$.

(b) The natural map $\mathbf{K}^{\beta} \rightarrow C$ is quasihomogeneous and $\mathcal{H}^{\beta}$-measure preserving but not bilipschitz.

(c) There are Möbius transformations $T_{k}: \overline{\mathbf{R}}^{2} \rightarrow \overline{\mathbf{R}}^{2}$ with $\operatorname{diam} T_{k}(C)=1$ for all $k$ yet either $\mathcal{H}^{\beta}\left(T_{k}(C)\right) \rightarrow 0$ or $\mathcal{H}^{\beta}\left(T_{k}(C)\right) \rightarrow \infty$.

Proof. Parts (a), (b) follow because $C$ has lower density zero; (c) is a consequence of [GH, Corollary 3.5].

\section{HOMEOMORPhisms of Jordan CuRves}

We conclude our work by comparing various classes of homeomorphisms defined on Jordan curves. Clearly bilipschitz maps are always both quasihomogeneous and (by Corollary 2.3) quasi-measure preserving (abbreviated here as QMP). We ask when are these maps bilipschitz, and what are the relations between the two classes QH and QMP? We make repeated use of Theorem D, however we delay its proof until the end of this section.

Verification of the following is left as an exercise for the interested reader.

6.1. Fact. The following are equivalent for self-homeomorphisms of the line $\mathbf{R}$ or the circle $\mathbf{S}^{1}: B L, Q H, Q M P$.

We take this opportunity to communicate the following which pertains to various parameterizations $\varphi: \mathbf{S}^{1} \rightarrow C$ for Jordan curves $C \subset \mathbf{R}^{n}$. These characterize information about $C$ in terms of conditions on the maps. To simplify our statements we say that $C$ is $\mathcal{H}^{\alpha}$-homogeneous if each non-degenerate subarc of $C$ has positive finite Hausdorff measure. Also, the notation $\mathrm{CA}^{\alpha}$ stands for an $\alpha$-dimensional chordarc condition which we examined in detail in $[\mathrm{GH}]$. 
6.2. Proposition. Let $C \subset \mathbf{R}^{n}$ be a Jordan curve. Then:

(a) $C$ is $\mathcal{H}^{\alpha}$-homogeneous if and only if there is a $Q M P \varphi: \mathbf{S}^{1} \rightarrow C$.

(b) $C$ is $C A$ if and only if there is a $B L \varphi: \mathbf{S}^{1} \rightarrow C$.

(c) $C$ is $C A^{\alpha}$ if and only if there is a $B L^{\alpha} \varphi: \mathbf{S}^{1} \rightarrow C$.

(d) $C$ is $B T$ if and only if there is a $Q S \varphi: \mathbf{S}^{1} \rightarrow C$.

(e) $C$ is BLH and BT if there is a $Q H \varphi: \mathbf{S}^{1} \rightarrow C$, and there exists such a $\varphi$ if in addition $C$ is an $\alpha$-set.

Proof. Part (a) follows from Fact 2.1. Parts (b), (d) are well known and (c) was proved in [GH, Theorem B]. Part (e) is essentially Theorem C.

Now we explain how (e) above is best possible. First, there are $\mathrm{QH} \varphi: \mathbf{S}^{1} \rightarrow C$ with $C$ having zero Hausdorff measure; also, we can get such $\varphi: \mathbf{S}^{1} \rightarrow C$ with each non-degenerate subarc of $C$ having infinite Hausdorff measure. See Example 5.3. Next, in [GH, Example 5.4] we constructed 'nice' BT curves which are not BLH. Thus there are examples of measure preserving QS $\varphi: \mathbf{S}^{1} \rightarrow C$ which are not QH. On the other hand, according to Theorem D, if $C$ is a 'nice' BLH BT curve, then a parameterization $\varphi$ of $C$ is QMP if and only if it is QH. We hasten to point out that Theorem E yields an example of a 'nice' BLH BT curve which does not have a BL ${ }^{\alpha}$ parameterization. Finally, in light of the above, we mention that when $C \subset R^{n}$ is $\mathrm{BT}$ and $\varphi: \mathbf{S}^{1} \rightarrow C$ is QMP, then $\varphi$ is QH if and only if $C$ is BLH.

Next we consider constraints on $C$ which permit us to assert that a $\mathrm{QH}$ homeomorphism $\varphi: \mathbf{S}^{1} \rightarrow C$ is $\mathrm{BL}$ or $\mathrm{BL}^{\alpha}$. Again we mention that Theorem E produces an example of an $\mathcal{H}^{\beta}$-rectifiable BLH BT curve, with a $\mathrm{QH}$ parameterization, which does not have a $\mathrm{BL}^{\beta}$ parameterization.

6.3. Corollary. Let $\varphi: \mathbf{S}^{1} \rightarrow C$ be $Q H$. Then $C$ is rectifiable if and only if $\varphi$ is $B L$ and $C$ is $C A^{\alpha}$ if and only if $\varphi$ is $B L^{\alpha}$.

Proof. According to Theorem B, the first assertion is a consequence of the second. The necessity of the latter claim follows from Proposition 6.2(c) since $C$ being $\mathrm{CA}^{\alpha}$ means there exist a BL ${ }^{\alpha}$ (hence QH) $\psi: \mathbf{S}^{1} \rightarrow C$, so $h=\psi^{-1} \circ \varphi: \mathbf{S}^{1} \rightarrow \mathbf{S}^{1}$ is $\mathrm{QH}$, hence BL by Fact 6.1 , and thus $\varphi=\psi \circ h$ is the composition of a $\mathrm{BL}^{\alpha}$ map and a BL map.

An interesting phenomenon occurs for self-homeomorphisms: Aseev and Shalaginov demonstrated that QH self-maps of continua are BL [AS, Theorem 2].

Finally, we turn our attention to homeomorphisms between two Jordan curves. Before validating Theorem $\mathrm{D}$, we mention that it is possible to have a QMP QH homeomorphism between two BLH BT Jordan curves which is not $\mathrm{BL}^{\alpha}$, even when the curves are of the same dimension and have positive finite Hausdorff measure; e.g., consider a measure preserving map between the Mattila snowflake constructed in Theorem $\mathrm{E}$ and the appropriate dimension von Koch snowflake curve- by Theorem D this map is QH, but it cannot be BL because the Mattila snowflake has lower $\mathcal{H}^{\alpha}$-density zero everywhere and hence is not $\mathrm{CA}^{\alpha}$. On the other hand, when both curves are rectifiable (i.e., one-dimensional), any QMP or QH map between them must be BL.

Proof of Theorem D. Let $h$ be a homeomorphism between two BLH BT Jordan curves $C \subset \mathbf{R}^{n}, C^{\prime} \subset \mathbf{R}^{m}$ which are $\alpha$-, $\beta$-sets respectively. Let $\varphi, \psi$ be the $\mathcal{H}^{\alpha_{-}}$, $\mathcal{H}^{\beta}$-parameterizations respectively. Then by the proof of Theorem 4.6 we know that $\varphi$ and $\psi$ are both QH. Thus if $h$ is QMP (QH), then so is $g=\psi^{-1} \circ h \circ \varphi: \mathbf{S}^{1} \rightarrow \mathbf{S}^{1}$, 
therefore by Fact $6.1 \mathrm{~g}$ is BL, and hence $h=\psi \circ g \circ \varphi^{-1}$ is QH (QMP). It is not hard to see that the parameters for $h$ depend only on each other and the data for $C$ and $C^{\prime}$.

\section{Questions}

1. What is the analog of Theorem A for curves $C$ in $\mathbf{R}^{n}$ which are homogeneous with respect to $\mathcal{B} \mathcal{L}_{K}\left(\mathbf{R}^{n}\right)$ ?

2. Is there a bilipschitz homogeneous curve which is not bounded turning?

3. Are bilipschitz homogeneous curves in $\mathbf{R}^{2}$ quasicircles? Which quasicircles in $\mathbf{R}^{2}$ are bilipschitz homogeneous?

4. What are the relations among the constants $K, a, \alpha$ for $K-\mathrm{BLH} a-\mathrm{BT}$ curves of dimension $\alpha$ ?

Added in proof. Further work on bilipschitz homogeneity can be found in a forthcoming paper by the second author and Volker Mayer.

\section{REFERENCES}

[AS] V.V.Aseev and A.A.Shalaginov, Mappings that boundedly distort distance ratios, Russian Akad. Sci. Dokl. Math. 49 (1994) 248-250. MR 96a:30019

[BE] B. Brechner and T. Erkama, On topologically and quasiconformally homogeneous continua, Ann. Acad. Sci. Fenn. Ser. A I Math. 4 (1978/79) 207-208. MR 81c:54051

[E1] T. Erkama, Quasiconformally homogeneous curves, Michigan Math. J. 24 (1977) 157159. MR 57:6417

[E2] T. Erkama, On domains of bounded dilatation, pp.68-75, Proceedings 5th RomanianFinnish Seminar in Complex Analysis, Lecture Notes in Math., No. 1013, SpringerVerlag, Berlin, 1983. MR 85j:30038

[F1] K.J.Falconer, The Geometry of Fractal Sets, Cambridge Univ. Press, Cambridge, 1986. MR 88d:28001

[F2] K.J.Falconer, Fractal Geometry: Mathematical Foundations and Applications, John Wiley and Sons Ltd., West Sussex, England, 1990. MR 92j:28008

[GP] F.W.Gehring and B.P.Palka, Quasiconformally homogeneous domains, J. Analyse Math. 30 (1976) 172-199. MR 55:10676

[G] M.Ghamsari, Quasiconformal Fuchsian groups acting on $B^{3}$ that are not quasiconformal conjugate to Möbius groups, Ann. Acad. Sci. Fenn. Ser. A I Math. 20 (1995) 245-250. MR 96h:30034

[GH] M.Ghamsari and D.A.Herron, Higher dimensional Ahlfors regular sets and chordarc curves in $\mathbf{R}^{n}$, Rocky Mountain J. Math., to appear.

$[\mathrm{H}] \quad$ J.E. Hutchinson, Fractals and self similarity, Indiana Univ. Math. J. 30 (1981) 713747. MR 82h:49026

[LV] O. Lehto and K.I. Virtanen, Quasiconformal Mappings in the Plane, Springer-Verlag, Berlin, 1973. MR 52:14282

[MNP] P.MacManus, R.Näkki and B.P.Palka, Quasiconformally homogeneous compacta in the complex plane, Michigan Math. J. 45 (1998) 227-241. CMP 98:16

[Mt] P.Mattila, Geometry of Sets and Measures in Euclidean Spaces: Fractals and Rectifiability, Cambridge studies in advanced math., 44, Cambridge Univ. Press, Cambridge, 1995. MR 96h:28006

[My] V.Mayer, Trajectoires de groupes à 1-paramètre de quasi-isométries, Revista Mat. Iber. 11 (1995) 143-164. MR 96f:28005

[Sa] J. Sarvas, Boundary of a homogeneous Jordan domain, Ann. Acad. Sci. Fenn. Ser. A I Math. 10 (1985) 511-514. MR 86m:30021

[Sh] A.A.Shalaginov, On mappings of self-similar curves, Siberian Math. J. 34 (1993) 11901195. MR 95g:30027

[T] P.Tukia, A quasiconformal group not isomorphic to a Möbius group, Ann. Acad. Sci. Fenn. Ser. A I Math. 6 (1981) 149-160. MR 83b:30019 
[TV1] P.Tukia and J.Väisälä, Quasisymmetric embeddings of metric spaces, Ann. Acad. Sci. Fenn. Ser. A I Math. 5 (1980) 97-114. MR 82g:30038

[TV2] P.Tukia and J.Väisälä, Bilipschitz extensions of maps having quasiconformal extensions, Math. Ann. 269 (1984) 561-572. MR 86c:30041

[V] J.Väisälä, Quasiconformal maps of cylindrical domains, Acta Math. 162 (1989) 201225. MR 90f:30034

Department of Mathematics, University of Cincinnati, Cincinnati, Ohio 45221

E-mail address: manouchehr.ghamsari@ucollege.uc.edu

Department of Mathematics, University of Cincinnati, Cincinnati, Ohio 45221-0025

E-mail address: david.herron@math.uc.edu 